\title{
Surveillance of infant pertussis in Sweden 1998-2012; severity of disease in relation to the national vaccination programme
}

R M Carlsson (rose-marie.carlsson@vgregion.se) ${ }^{1,2}$, K von Segebaden ${ }^{1}$, J Bergström ${ }^{1}$, A M Kling ${ }^{1}$, L Nilsson ${ }^{1,3}$

1. The Public Health Agency of Sweden, Stockholm, Sweden

2. Unit of Infection Control, Department of Clinical Microbiology, Sahlgrenska University Hospital, Gothenburg, Sweden

3. Allergy Centre, Clinical and Experimental Medicine, Linköping University, Linköping, Sweden

Citation style for this article:

Carlsson RM, von Segebaden K, Bergström J, Kling AM, Nilsson L. Surveillance of infant pertussis in Sweden 1998-2012; severity of disease in relation to the national vaccination programme. Euro Surveill. 2015;20(6):pii=21032. Available online: http://www.eurosurveillance.org/ViewArticle.aspx?Articleld=21032

In Sweden, pertussis was excluded from the national vaccination programme in 1979 until acellular vaccination was introduced in a highly endemic setting in 1996. The general incidence dropped 10 -fold within a decade, less in infants. Infant pertussis reached 40-45 cases per 100,000 in 2008 to 2012; few of these cases were older than five months. We present an observational 15-year study on the severity of infant pertussis based on 1,443 laboratory-confirmed cases prospectively identified from 1998 to 2012 in the national mandatory reporting system and followed up by telephone contact. Analyses were made in relation to age at onset of symptoms and vaccination history. Pertussis decreased in non-vaccinated infants (2003 to 2012, $\mathrm{p}$ < 0.001), indicating herd immunity, both in those too young to be vaccinated and those older than three months. The hospitalisation rates also decreased (last five-year period vs the previous five-year periods, $p$ <0.001), but $70 \%$ of all cases in under three month-old infants and $99 \%$ of cases with apnoea due to pertussis were admitted to hospital in 1998 to 2012. Median duration of hospitalisation was seven days for unvaccinated vs four days for vaccinated infants aged $3-5$ months. Nine unvaccinated infants died during the study period.

\section{Introduction}

An increase in pertussis incidence, with large outbreaks, has been observed in recent years in many high-income countries despite high vaccination coverage rates. These epidemiological changes are mainly seen in adolescents, adults and infants too young to be vaccinated, with the most severe morbidity in the latter group. In response to a high rate of pertussis in infants, several countries have considered or adopted additional strategies for improved pertussis control in this age group, with the main focus on preventing severe disease and death in the youngest infants.

In this age group, the options are to induce immunity in the infant by optimising the vaccination schedule and adherence, to prevent transmission of Bordetella pertussis to the infant from close contacts and/or others in the community, and to reduce the effect by post-exposure chemoprophylaxis after transmission has occurred, or a combination of these primary and secondary prevention strategies. Whatever strategy is chosen, there is a need to monitor the effect in the target group over time.

In Sweden, the national vaccination programme (NVP) has included infant vaccination against pertussis at 3, 5 and 12 months of age since 1996. From 2007, an early school booster vaccination has been added, and already in 1982, post-exposure chemoprophylaxis was recommended to infants exposed to pertussis. The epidemiology of pertussis is monitored through regular surveillance and a prospective long-term enhanced pertussis surveillance project [1].

In this study of infant pertussis, markers of severity were related to age at onset of disease and to individual vaccination history as well as to the scheduled ages of the NVP. We present age-specific complication and hospitalisation rates in vaccinated and unvaccinated infants, with information on duration of hospital stay, and also the relation between early or delayed onset of antibiotic treatment and duration of cough in infants.

\section{Methods}

This observational study of pertussis in infants encompasses information obtained in two ways: within the regular Swedish surveillance of communicable diseases and from a long-term enhanced pertussis surveillance study.

\section{Regular surveillance}

In Sweden, pertussis is one of ca 60 notifiable diseases, and one of ca 40 diseases with mandatory contact tracing. Both clinicians and laboratories report any suspected or confirmed case by notifications both to the Public Health Agency of Sweden and to the County 
Medical Officer in Communicable Disease Prevention and Control. The notifications are immediately available at both national and regional level through a webbased registry (SmiNet) based on disease and personal identifiers [2]. There is little or no information in the reports on vaccination status or clinical details including case contacts, but age-specific incidence rates can be calculated from age at reporting date (as a surrogate for age at onset of disease).

A suspected case in Sweden is defined by clinical signs compatible with pertussis plus an epidemiological link, whereas a confirmed case is a case with a positive culture, PCR or serology (seroconversion or significant increase in IgG against pertussis toxin) [3]. Notably, $P C R$ has been increasingly used in the past decade, with culture-confirmed pertussis becoming rare.

\section{Enhanced surveillance}

The enhanced surveillance of pertussis in Sweden was established in October 1997 and is still ongoing. Every case of laboratory-reported pertussis in children born in 1996 or later is identified in SmiNet for detailed follow-up, with the exception until 2003 of cases in one area where a local surveillance project was in place [4]. The present analysis includes all infant cases identified from start of the project through 2012. The number of live births in Sweden ranged from 90,502 in 1997 to 113,177 in 2012.

All identified infant reports were matched against the population registry for parental contact details and to check that there was no death notification. A research nurse performed structured telephone interviews with the parents of each case, using a standardised questionnaire. The clinical questions included type and duration of cough, presence of apnoea and other complications, number and length of hospital admissions, and timing of antibiotic treatment if given. The nurse also contacted the child healthcare centre (CHC) of every infant to obtain documented vaccination dates, products and batch numbers. Families of deceased infants were not contacted, but their $\mathrm{CHC}$ provided vaccination status, and information on gestational age was obtained from the medical birth register. Infants were also excluded from detailed follow-up if an interview was impossible due to language problems or if the family could not be found.

The individual vaccination history allowed for calculation of age-specific incidence rates in vaccinated and non-vaccinated children. Because of the clinical information, these calculations were based on age at onset of symptoms, which is important when analysing agespecific severity of disease in infants.

Clinical data were analysed according to several cough definitions, including 14 days of coughing corresponding to the current case definitions for surveillance for the European Union (EU) [5] and from the World Health Organization (WHO) [6] and 21 days of paroxysmal cough according to a previous WHO definition established for use in efficacy trials.

\section{Statistics}

Population data used when calculating the incidences were obtained from Statistics Sweden (www.scb.se).

A test for time trend of the incidence was calculated using a quasi-Poisson regression model, used due to overdispersion. Interquartile range (IQR) was used to describe variability in duration of hospital stay. Fisher's exact test was used for comparison of proportions.

\section{FIGURE 1}

Pertussis incidence according to regular notifications of laboratory-confirmed pertussis, Sweden, 1986-2012

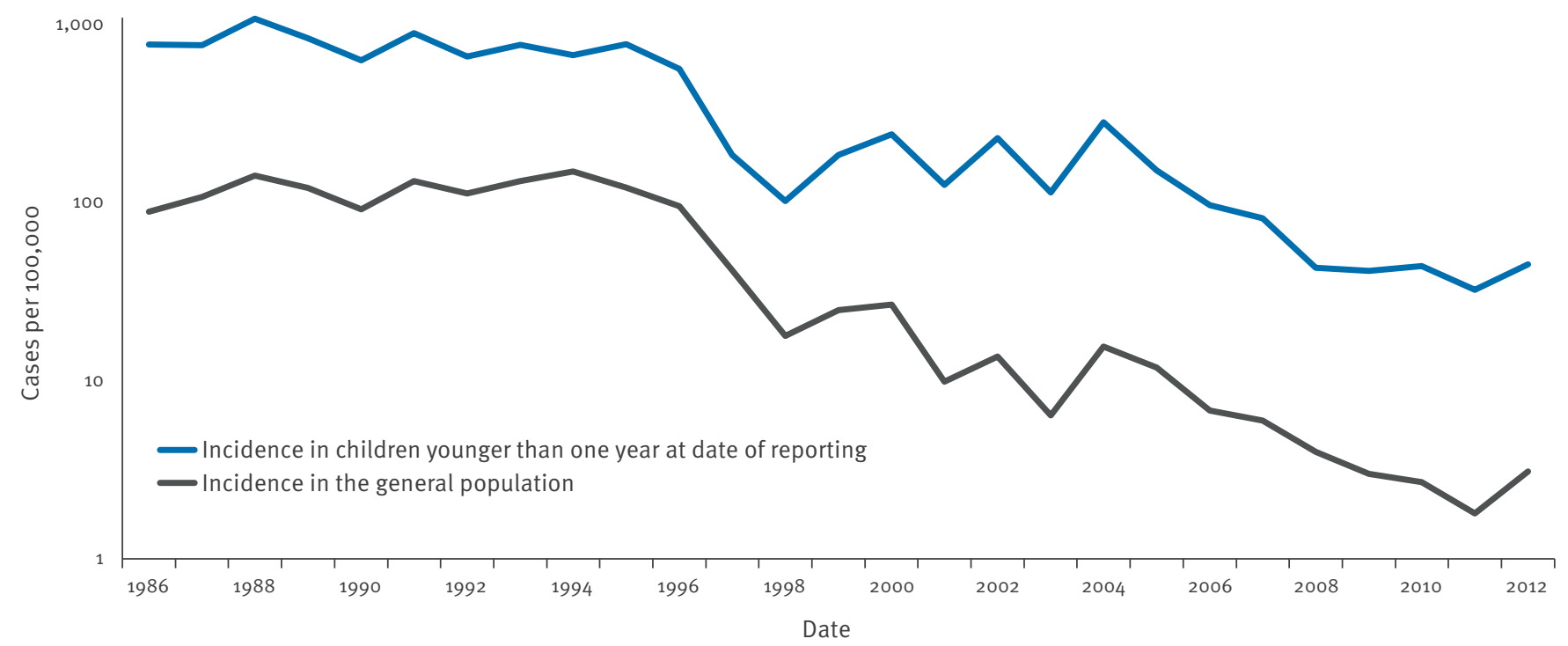




\section{Results}

\section{Regular surveillance}

Figure 1 illustrates the incidence of laboratory-confirmed pertussis in the whole Swedish population and in infants, before and after introduction of the acellular vaccination in 1996. During the first decade of vaccination there was a marked initial drop in disease incidence followed by a more stable period albeit with remaining epidemic cycles. During the last five-year period of the study there was again an initial drop followed by a more stable period, this time without substantial variation in incidence from year to year. Throughout the 15 years 1998 to 2012, the incidence in the general population decreased more or less continuously from ca 90 per 100,000 before 1996 to less than 10 per 100,000 in 2006 and onwards. The incidence in infants also decreased, but more slowly and more stepwise, from over 500 per 100,000 in 1996 to less than 50 per 100,000 during the last five of the 15 studied years.

\section{Enhanced surveillance}

From October 1997 through 2012, there were 1,803 laboratory-reported cases of pertussis in infants in Sweden. The families of nine deceased infants were not contacted for ethical reasons, 315 infant cases between 1997 and 2002 occurred in the area not included in the enhanced surveillance at the time and were therefore non-eligible, and 36 families were excluded due to lack of contact details or language problems. Vaccination history and information on cough and antibiotic treatment was collected for all remaining 1,443 cases, including date of onset of coughing. Data on complications or hospitalisation were available for 1,426 of the 1,443 of the cases.

Overall, 840 infants were unvaccinated and 603 had received at least one vaccine dose against pertussis by the time of disease onset. Among the unvaccinated cases, 698 were younger than the scheduled age of three months for the first pertussis vaccine dose. Approximately one quarter of infants aged 3-5 months $(104 / 395,26 \%)$ had not received their first scheduled dose and approximately one third of infants aged 5-12 months $(121 / 348,35 \%)$ had received only one but not both of the recommended doses at three and five months of age.

The difference between the date of first symptoms and the date of laboratory reporting (which also is the date of the laboratory analysis) ranged from -12 to 93 days, with a median of 12 days. The negative value means that some laboratory samples were drawn in asymptomatic infants in the context of contact investigation.

Time trend for incidence in infancy

There was a high incidence of pertussis with onset of symptoms during the first four or five months of life, followed by a steep decline in infants six months and older. The incidence of pertussis was 150, 208 and 193 per 100,000 person years for the first, second and third month of infancy, respectively, but with a successive decrease over the years after vaccination was introduced. This time trend is illustrated in Figure 2.

\section{FIGURE 2}

Age-specific number of laboratory-confirmed pertussis cases per 100,000 infants, Sweden, 1998-2012 (n=1,418 infants in enhanced surveillance project)

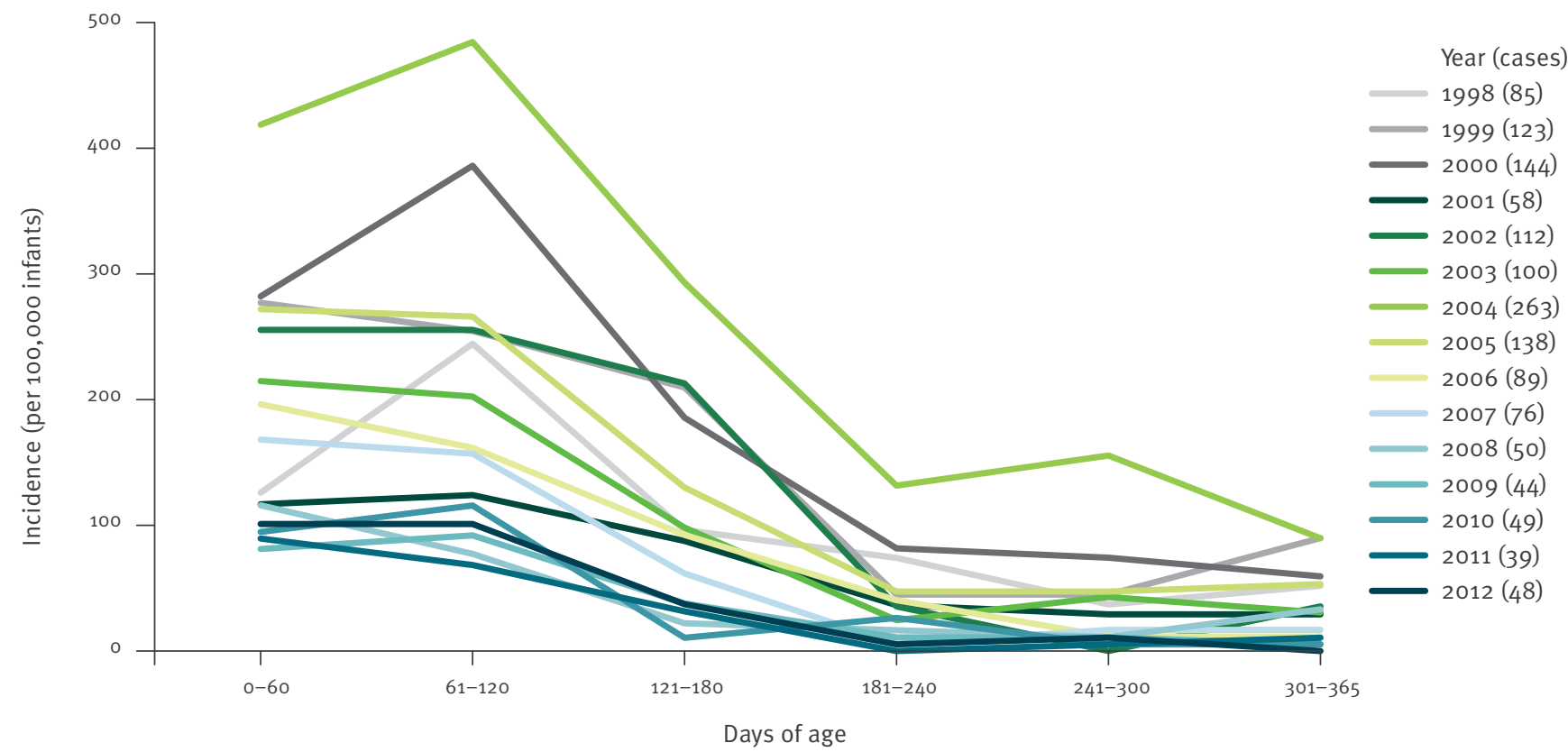


Reported pertussis in non-vaccinated infants decreased in the period 2003 to 2012 when comparing to the previous five-year period ( $p<0.001$ for infants $\leq 90$ days: odds ratio $(\mathrm{OR})=0.36 ; 95 \%$ confidence interval $(\mathrm{Cl})$ : $0.29-0.44 ; \mathrm{p}<0.001$ for infants $>90$ days: $O R=0.33$; $95 \% \mathrm{Cl}: 0.18-0.58)$. This was seen both in infants younger than three months, too young to be vaccinated, and in those who were unvaccinated although they were between three and 12 months-old.

\section{Deaths}

There were nine infant deaths during the first 11 years of the surveillance period. All were unvaccinated and all were healthy before falling ill with pertussis. Eight infants died at the age of 1-4 month and one at six months of age. Five were full term whereas four were born before gestational week 37 . The child who died aged six months was extremely preterm with a birth weight of $630 \mathrm{~g}$.

An estimate of the case fatality rate can be made from age at laboratory report of pertussis in relation to the total number of laboratory-confirmed pertussis cases in children younger than six months at date of laboratory report. On this basis, the case fatality rate in this age group was $0.65 \%(9 / 1,375)$.

\section{Duration of cough}

All but 36 infants had a cough of at least two weeks $(1,407 / 1,443 ; 98 \%)$. Among the 36 infants, coughing lasted for a range of zero to 13 days, and the cough was of paroxysmal type in 15 cases. Six of the 36 infants were hospitalised.

Among 700 cases younger than three months, 599 (86\%) had a paroxysmal cough of 21 days or more. In 1,281 of all 1,443 infants (89\%) paroxysmal cough was observed, which lasted for 21 days or longer in 1,151 infants (79.8\%).

\section{Complications}

The complication rate was $41 \%$ (287/694) in infants younger than three months and $16 \%$ (116/732) in infants aged 3-12 months (Table 1). In children younger than three months who suffered from apnoea, $99 \%$ (153/155) were hospitalised. In the age group 3-12 months, seven of eight unvaccinated children with dehydration were hospitalised compared with 12 of 21 vaccinated children $(p=0.201)$.

Hospitalisation rates in vaccinated and non-vaccinated infants

Hospital admission rates in infants aged $0-2,3-4$ and 5-12 months at onset of disease were 131, 58 and 5 per 100,000 person-years, respectively. The incidence in the youngest infants aged 0, 1 or 2 months was, respectively, 127, 152 and 114 per 100,000. The proportions of laboratory-confirmed cases that were hospitalised in the same age groups were 82,71 , and $57 \%$. In the two older age groups 3-4 and 5-12 months, the proportions were 36 and $12 \%$, respectively. Figure $3 \mathrm{~A}$ compares incidence of laboratory-confirmed pertussis in these age groups with the hospital admission incidence rates per 100,000 infants. The younger the infant, the higher the proportion of cases hospitalised. In the youngest group (o-30 days) the confidence intervals overlapped, indicating that almost all cases were hospitalised. The peak of incidence and of hospitalisations was during the second month of life.

In Figure $3 \mathrm{~B}$ we have plotted the hospital admission rates per 100,000 person-years during the three fiveyear periods of 1998-2002, 2003-07 and 2008-12. We found a similar age pattern over these three time periods, with slightly higher incidences during the second compared with the first period because of a large outbreak in 2004. The incidence was lower during the third five-year period.

Unvaccinated infants younger than five months were discharged after a median of seven days (IQR: 4-12 days for infants younger than three months; IQR: 3-13 days for 3-5 month-old infants), whereas the vaccinated infants aged 3-5 months had a median duration of hospital stay of four days (IQR 2-8 days). All infants five months or older were discharged after a median of 3.5 days (unvaccinated IQR: 2-14 days, vaccinated IQR: 2-5 days).

\section{TABLE 1}

Hospitalisation rates among infants with $(n=403)$ and without $(n=1,023)$ complications due to pertussis, by age group and vaccination status (at least one dose), Sweden, 1998-2012

\begin{tabular}{|c|c|c|c|c|c|c|c|c|c|c|}
\hline & \multicolumn{4}{|c|}{$\begin{array}{l}\text { Hospitalisation rates in } \\
\text { infants }<3 \text { months }\end{array}$} & \multicolumn{4}{|c|}{$\begin{array}{l}\text { Hospitalisation rates in } \\
\text { infants } 3^{-12} \text { months }\end{array}$} & \multirow{2}{*}{\multicolumn{2}{|c|}{$\begin{array}{l}\text { Hospitalisation rates } \\
\text { in all infants }\end{array}$}} \\
\hline & \multicolumn{2}{|c|}{ Unvaccinated } & \multicolumn{2}{|c|}{ Vaccinated } & \multicolumn{2}{|c|}{ Unvaccinated } & \multicolumn{2}{|c|}{ Vaccinated } & & \\
\hline & $\mathrm{n} /$ total & $\%$ & $\mathrm{n} /$ total & $\%$ & $\mathrm{n} /$ total & $\%$ & $\mathrm{n} /$ total & $\%$ & $\mathrm{n} /$ total & $\%$ \\
\hline Apnoea & $152 / 154$ & 99 & $1 / 1$ & 100 & $9 / 10$ & 90 & $27 / 32$ & 84 & $189 / 197$ & 96 \\
\hline Breathing problems & $75 / 76$ & 99 & $1 / 1$ & 100 & $15 / 19$ & 79 & $16 / 25$ & 64 & $106 / 121$ & 88 \\
\hline Dehydration & $48 / 53$ & 91 & $0 / 0$ & 0 & $7 / 8$ & 88 & $12 / 21$ & 57 & $67 / 82$ & 82 \\
\hline Seizures & $1 / 1$ & 100 & $0 / 0$ & 0 & $1 / 1$ & 100 & $0 / 0$ & 0 & $2 / 2$ & 100 \\
\hline All complications & $276 / 285$ & 97 & $2 / 2$ & 100 & $32 / 38$ & 84 & $55 / 78$ & 71 & $365 / 403$ & 91 \\
\hline No complications & $206 / 407$ & 51 & $0 / 0$ & 0 & $26 / 101$ & 26 & $71 / 515$ & 14 & $303 / 1,023$ & 30 \\
\hline
\end{tabular}




\section{FIGURE 3}

Laboratory-confirmed pertussis cases per 100,000 infants, 1 October 1997-31 December $2012(\mathrm{n}=1,443$, whereof $\mathrm{n}=667$ hospitalised) and hospitalised cases per 100,000 infants, 1998-2002, 2003-07 and 2008-12, Sweden

A. Incidence of pertussis by age group

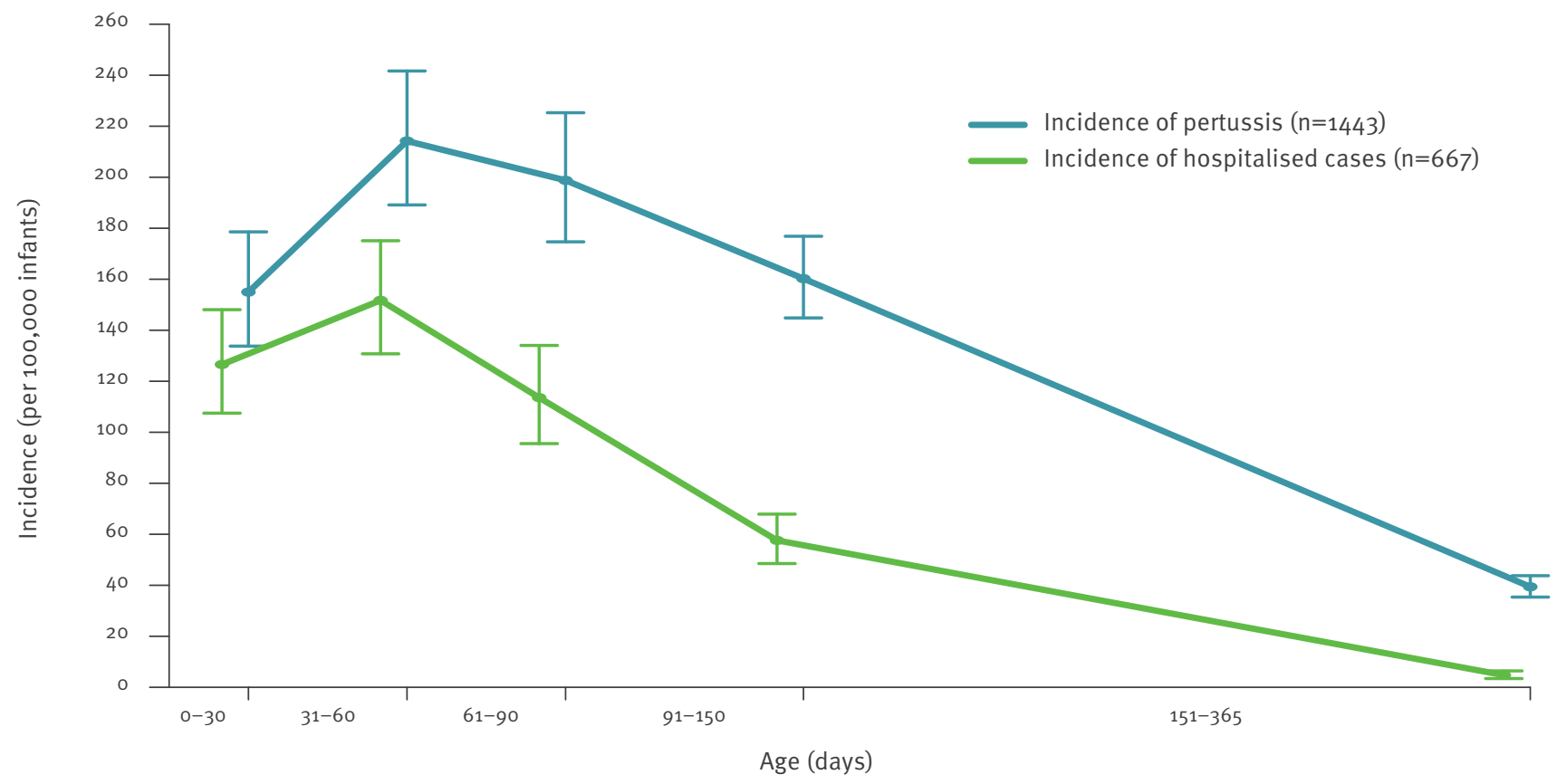

B. Incidence of hospitalisations by time period

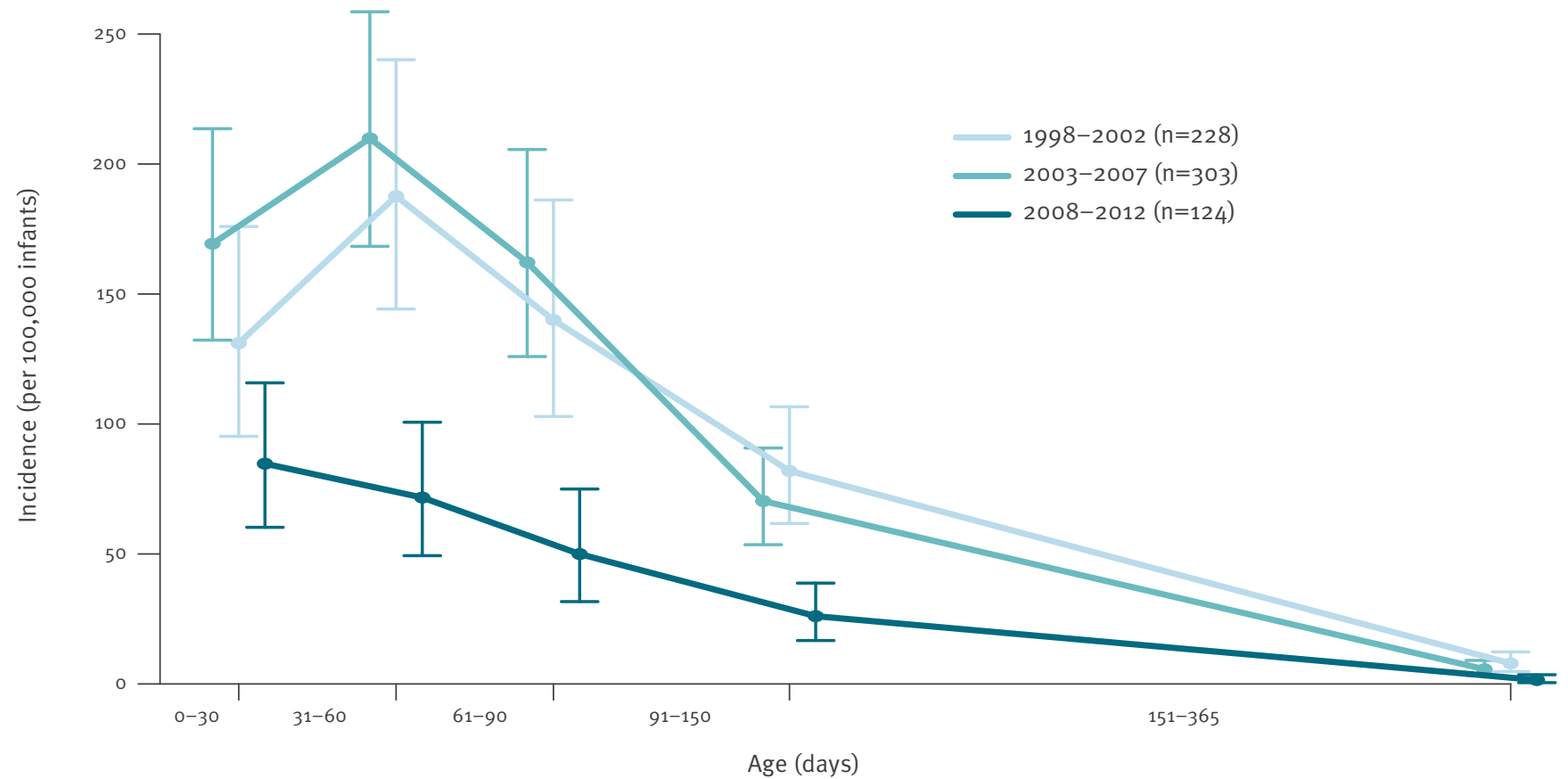

Age is calculated at date of onset of disease and the age intervals during the infant year are chosen in relation to the vaccination schedule at age 3,5 and 12 months (0-90, 91-150 and 151-365 days), with the 0-90-day interval further divided in months (0-30, 31-60 and 61-90 days). 
Antibiotic treatment

Antibiotics were prescribed to 1,233 of 1,443 (85\%) of the infants, of whom 1,109 ( $77 \%$ ) had paroxysmal cough Figure 4. We compared the median durations of cough (in days) by Fisher's exact test. Doing this pairwise in the group aged 0-90 days and the group aged 91-365 days, an early start of the antibiotic treatment, within the first week ( $\leq 6$ days) after onset of cough during the episode was shown to be associated, in all age groups, with a shorter duration of the coughing period compared with those who had antibiotic treatment initiated later than two weeks after onset of cough or no treatment (Table 2).

Furthermore, antibiotics were prescribed to 33 of 36 infants with cough duration of less than two weeks, and most of these (26/32) had an early start of the antibiotic treatment. This is in contrast to a late start in the vast majority $(1,033 / 1,192)$ of cases with at least 14 days of cough; among those, the antimicrobial therapies were initiated during the first week in only $13 \%$ (79/619) of infants younger than three months, and in $14 \%(80 / 573)$ of infants aged 3-12 months.

\section{Discussion}

The paper describes data on severity of pertussis in Swedish infants during the first 15 years of an acellular vaccination programme, as reflected through regular and enhanced surveillance.
Regular surveillance data indicate a dramatic decrease in laboratory-confirmed pertussis in the first 10 years after the introduction of pertussis vaccination in the NVP in 1996 [7]. There was a steep initial decline in both the general populations and in infants. The incidence decreased further in the general population, while infant pertussis continued to oscillate above the level of 100 per 100.000 , with a large outbreak in 2004. Because there were signs of waning immunity six or seven years after vaccination in infancy [8], a pre-school booster was introduced at the age of six years in 2007 , with a catch-up at the age of 10 years. During the subsequent period 2008-12, pertussis incidence decreased further. Infant pertussis settled at a low level of ca 40-45 per 100,000, with no major oscillations, and the overall level in the population at between two and four cases per 100,000.

This stepwise achievement of pertussis control through acellular vaccination is unique for Sweden because all other countries introduced these vaccines in an already controlled situation by changing from whole-cell vaccination. Interestingly, epidemiological data from the United Kingdom (UK) after 1957, when whole-cell pertussis vaccines were introduced in a non-vaccinating and highly endemic setting [9], are compatible with the Swedish data after 1997, when acellular pertussis vaccines were introduced in a similar situation. However, it is too early to tell if the acellular vaccines also in the

\section{FIGURE 4}

Duration of paroxysmal cough in infants with laboratory-confirmed pertussis, in relation to antibiotic treatment and to age, Sweden, 1998-2012 ( $\mathrm{n}=1,443)$
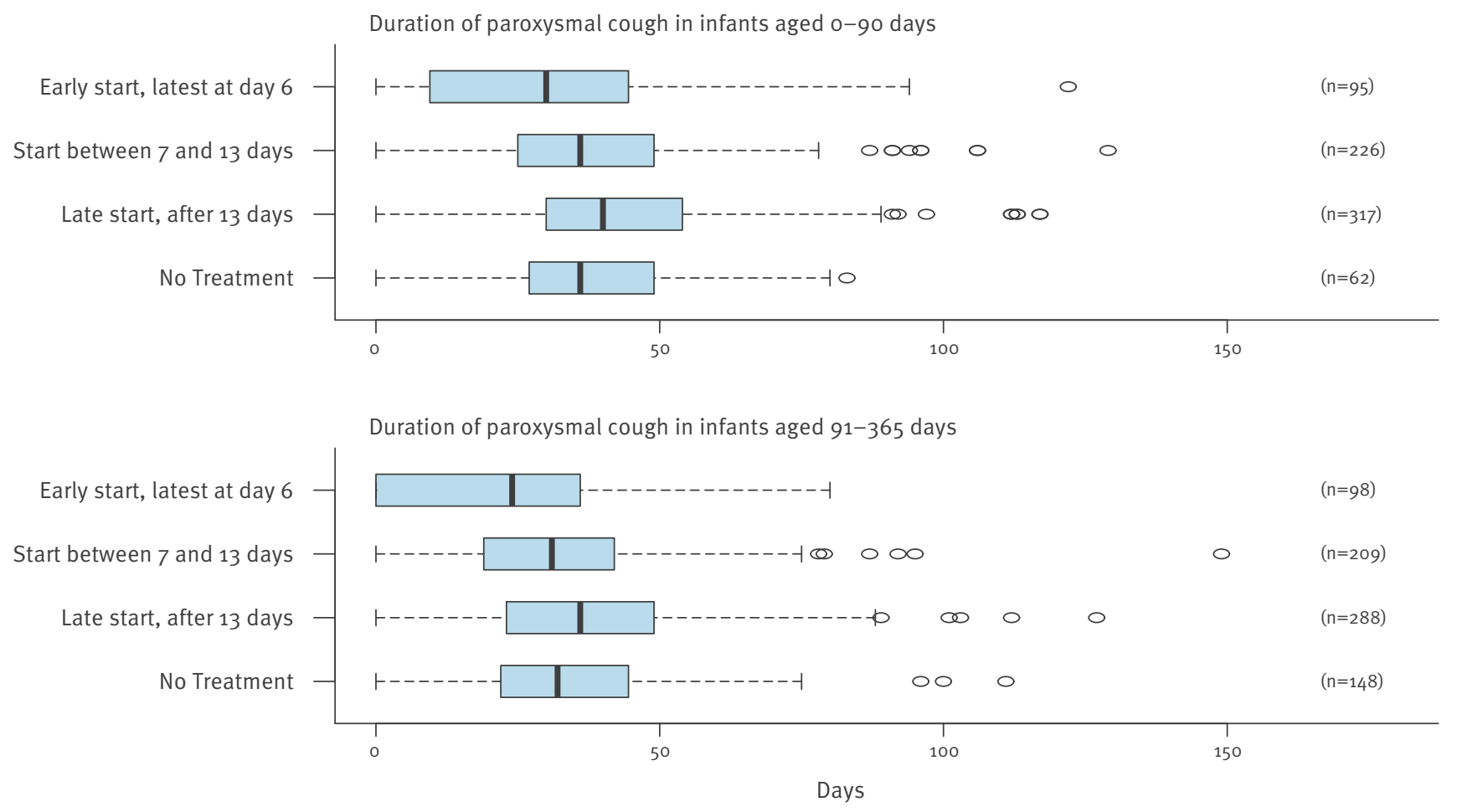

Boxes show first and fourth quartile, whiskers extend to the most extreme point.

Antibiotic treatment was with erythromycin or trimethoprim-sulfametoxazol. 
longer run will result in an epidemiological situation similar to when whole cell vaccines are used.

The data from the enhanced surveillance reveal that over the 15 -year study period, the age-specific incidence rates decreased also in non-vaccinated infants too young to be vaccinated and in those who were unvaccinated despite their age of 3-12 months. These results indicate indirect protection from reduced exposure. With no cocooning strategy or vaccination during late pregnancy implemented in Sweden, it seems likely that the explanation is a general decrease in the circulation of $B$. pertussis. Seroprevalence data comparing 1997 and 2007 are also supportive of this assumption [10].

There was also a significant reduction in hospitalisation rates in the period 2008 to 2012. A similar timetrend has been reported from the UK and from the Netherlands, with decreasing hospital rates noted four or five years after introduction of a pre-school booster $[11,12]$.

The hospital admission rates in Swedish infants were generally lower than those reported from the UK or the Netherlands $[11,12]$. The true hospitalisation rates in Sweden may be underestimated, as the trigger point for investigation was a positive laboratory sample. Others have demonstrated that linking of datasets may detect additional infants with hospital admissions, including presentation to emergency rooms [13]. This capturerecapture analysis is yet to be done in Sweden.

There was a peak in pertussis during second month of life, which is in accordance with other studies from the United States and Australia $[14,15]$. Their hospital admission rates, however, remained high also during third month of life in contrast to the marked reduction

\section{TABLE 2}

Comparison of duration of paroxysmal cough ( $p$ values) in relation to onset of antibiotic treatment in infants with laboratory-confirmed pertussis, Sweden, 1998-2012 $(\mathrm{n}=1,443)$

\begin{tabular}{|l|c|c|}
\hline \multirow{2}{*}{ Comparison } & \multicolumn{2}{|c|}{ Age group } \\
\cline { 2 - 3 } & $\leq 90$ days & $91-365$ days \\
\hline $\begin{array}{l}\text { Early start, latest at day 6 } \\
\text { vs Start between day 7 and 13 }\end{array}$ & 0.014 & 0.02 \\
\hline $\begin{array}{l}\text { Early start, latest at day 6 } \\
\text { vs Late start, after day 13 }\end{array}$ & 0.002 & $<0.001$ \\
\hline $\begin{array}{l}\text { Early start, latest at day 6 } \\
\text { vs No treatment }\end{array}$ & 0.024 & $<0.001$ \\
\hline $\begin{array}{l}\text { Start between day 7 and 13 } \\
\text { vs Late start, after day 13 }\end{array}$ & 0.258 & 0.001 \\
\hline $\begin{array}{l}\text { Start between day 7 and 13 } \\
\text { vs No treatment }\end{array}$ & $>0.999$ & 0.746 \\
\hline $\begin{array}{l}\text { Late start, after day 13 } \\
\text { vs No treatment }\end{array}$ & 0.129 & 0.107 \\
\hline
\end{tabular}

Four treatment categories were chosen and median durations of cough were compared pairwise by Fisher's exact test: (i) no treatment, (ii) early start ( $\leq 6$ days), (iii) start during second week (7-13 days) or (iv) late start ( $>2$ weeks) after onset of cough. in hospital stay observed in Swedish infants of this age. The discrepant observations may be a consequence of data sources and study methods, with our analyses related to age at onset of symptoms instead of age at hospital admission (or discharge).

It is well known that the highest complication and hospitalisation rates are found in infants too young to be vaccinated [16]. In the present study, a complication of any kind was reported in between one fourth and one third of the infants, and more frequently in unvaccinated infants. Respiratory distress, with or without apnoea, was the most frequent problem, and $97-99 \%$ of infants with breathing problems were admitted to hospital in the age group $0-3$ months. These findings are in accordance with 15-year data collected prospectively in the Swiss sentinel reporting system [17].

We have previously demonstrated some protection from the first dose of a pertussis vaccine, which significantly lowers incidence and hospitalisation rates in infants of the same age [18]. The present study confirms that vaccination reduces severity beyond what is attributable to age at onset of disease.

Post-exposure chemoprophylaxis to unvaccinated infants younger than six months was recommended in Sweden already in 1982 and so was early treatment to infants six to 12 months-old. These guidelines are still being followed: $91 \%$ of infants under the age of three months, and $78 \%$ of 3-11 month-old infants received antibiotics in the present study. However, these medications started two weeks after onset of symptoms in about half of the cases. This delay relates to the delay in case ascertainment, with a median of 11 days between the first day of symptoms and the day of laboratory confirmation. Unfortunately, we do not have information on the date of first medical visit. The research nurse who performed almost all of the 1,443 interviews, had the impression that many parents had to seek medical care several times before pertussis was suspected. Reasons seem to be that the baby was healthy between attacks of paroxysmal coughing and/ or that the medical personnel erroneously considered pertussis to be eliminated because of the NVP. Some parents complained that the doctors did not test for pertussis even in infants with typical pertussis, resulting in an unfortunate delay before proper diagnosis.

When treatment was initiated during first week of symptoms, the duration of paroxysmal cough was significantly shorter. These results partly contradict the general view that antibiotics usually have no effect on the course of the illness if given once the paroxysms are established [19], but are well in accordance with the opinion that antibiotics against pertussis limit the severity of disease if started in the catarrhal phase [20].

In our study, the general surveillance was able to indicate when control of pertussis was achieved by 
comparing the decrease of laboratory-reported pertussis in infants with that in the general population. But once the incidence of infant pertussis was at a low and stable level, only the enhanced surveillance could detect signs of herd immunity in the form of a significant decrease of pertussis in unvaccinated infants and also a significant decrease in hospitalisation rates. We conclude that there are two reasons to perform agespecific surveillance of pertussis in infants: to followup on vaccination effects in the priority target group, and because infants mirror the circulation of $B$. pertussis. How such surveillance is organised in detail will vary between countries, but detailed vaccination data and access to hospital admission data seem crucial, as well as monitoring of infant deaths due to pertussis.

In Sweden after 2009, there had not been any infant deaths due to pertussis until spring 2014, when a healthy full-term infant was in contact with two coughing parents already at birth. Unfortunately, pertussis was not considered and the infant died at three weeks of age from intractable pulmonary hypertension. In early summer 2014, another death occurred, also in a healthy full-term infant less than one month-old. Since the decrease in the circulation of $B$. pertussis means that mothers transfer lower concentrations of IgG antibodies against pertussis to their offspring [10], the risk of severe pertussis in the youngest infants may increase although their overall risk of contracting the disease is reduced. Careful surveillance, including agespecific data, will indicate if there is a need to consider complementary strategies such as maternal vaccination or cocooning.

Meanwhile, the present strategies to control infant pertussis could be improved, for instance through earlier primary prevention by adherence to the vaccination schedule, a higher degree of secondary prevention by increased awareness of symptoms compatible with the disease, and an earlier implementation of control measures around identified cases.

\section{Acknowledgments}

Financial support was obtained from the National Institute of Allergy and Infectious Diseases, Contract no. No1Al-15125, from the European Commission, Contract $n^{\circ}$ QLK2-CT-2001-01819; Eupertstrain, and from the following manufacturers an unrestricted grant: GlaxoSmithKline Vaccines, Wavre, Belgium, Sanofi Pasteur, Lyon, France, and Sanofi Pasteur MSD, Lyon, France.

\section{Conflict of interest}

None declared.

\section{Authors' contributions}

Rose-Marie Carlsson, who is a former head of the enhanced surveillance project, drafted and wrote the manuscript, and assisted in the analyses. Kerstin von Segebaden is the research nurse who called almost all 1443 families during
15 years, entering the data in the study database. Jakob Bergström and Anna-Maria Kling have performed the statistical analyses and commented on the manuscript. Lennart Nilsson, who is the current project leader, coordinated the work including the analyses, and assisted in writing.

\section{References}

1. Olin P, Gustafsson L, Barreto L, Hessel L, Mast TC, Rie AV, et al. Declining pertussis incidence in Sweden following the introduction of acellular pertussis vaccine. Vaccine. 2003;21(17-18):2015-21. http://dx.doi.org/10.1016/So264410X(02)00777-6 PMID:12706691

2. Rolfhamre P, Jansson A, Arneborn M, Ekdahl K. SmiNet-2: Description of an internet-based surveillance system for communicable diseases in Sweden. Euro Surveill. 2006;11(5):103-7. PMID:16757847

3. Falldefinitioner - vid anmälan enligt smittskyddslagen. [Case definitions - for notification under the Communicable Diseases Act]. Art.nr 2012-5-11. Stockholm: Socialstyrelsen; May 2012. Swedish. Available from: http://www.socialstyrelsen.se/Lists/ Artikelkatalog/Attachments/18700/2012-5-11.pdf

4. Trollfors B, Dotevall L, Sundh V, Welinder-Olsson C. Pertussis after end of a mass vaccination project--end of the "vaccination honey-moon". Vaccine. 2011;29(13):2444-50. http://dx.doi.org/10.1016/j.vaccine.2011.01.021 PMID:21292010

5. European Commission. Commission decision of 28 April 2008 amending Decision 2002/253/EC laying down case definitions for reporting communicable diseases to the Community network under Decision No 2119/98/EC of the European Parliament and of the Council. Official Journal of the European Union. 2008/426/EC. Available from: http://eur-lex.europa.eu/ LexUriServ/LexUriServ.do?uri=0J:L:2008:159:0046:0090:EN:P DF </eref>

6. World Health Organization (WHO). Generic protocol for estimating the burden of pertussis in young children. WHO/ IVB/05.15. Geneva: WHO: 2005. Available from: http:// whqlibdoc.who.int/hq/2005/WHO_IVB_05.15.pdf

7. Carlsson RM, Trollfors B. Control of pertussis--lessons learnt from a 10-year surveillance programme in Sweden. Vaccine. 2009;27(42):5709-18. http://dx.doi.org/10.1016/j. vaccine.2009.07.092 PMID:19679218

8. Gustafsson L, Hessel L, Storsaeter J, Olin P. Long-term follow-up of Swedish children vaccinated with acellular pertussis vaccines at 3,5 , and 12 months of age indicates the need for a booster dose at 5 to 7 years of age. Pediatrics. 2006;118(3):978-84. http://dx.doi.org/10.1542/peds.20052746 PMID:16950988

9. Amirthalingam G, Gupta S, Campbell H. Pertussis immunisation and control in England and Wales, 1957 to 2012: a historical review. Euro Surveill. 2013;18(38):20587. http://dx.doi.org/10.2807/1560-7917.ES2013.18.38.20587 PMID:24084340

10. Hallander HO, Andersson M, Gustafsson L, Ljungman M, Netterlid E. Seroprevalence of pertussis antitoxin (anti-PT) in Sweden before and 10 years after the introduction of a universal childhood pertussis vaccination program. APMIS. 2009;117(12):912-22. http://dx.doi.org/10.1111/j.16000463.2009.02554.x PMID:20078557

11. Campbell H, Amirthalingam G, Andrews N, Fry NK, George RC, Harrison TG, et al. Accelerating control of pertussis in England and Wales. Emerg Infect Dis. 2012;18(1):38-47. http://dx.doi. org/10.3201/eid1801.110784 PMID:22260989

12. van der Maas NAT, Mooi FR, de Greeff SC, Berbers GAM, Spaendonck MA, de Melker HE. Pertussis in the Netherlands, is the current vaccination strategy sufficient to reduce disease burden in young infants? Vaccine. 2013;31(41):4541-7. http:// dx.doi.org/10.1016/j.vaccine.2013.07.060 PMID:23933365

13. McCallum LK, Lio B, McIntyre P, Jorm LR. Estimating the burden of pertussis in young children on hopsitals and emergency departments: a study using linked routinely collected data. Epidemiol Infect. 2014;142(4):695-705.

14. Cortese MM, Baughman AL, Zhang R, Srivastava PU, Wallace GS. Pertussis hospitalizations among infants in the United States, 1993 to 2004. Pediatrics. 2008;121(3):484-92. http:// dx.doi.org/10.1542/peds.2007-1393 PMID:18310196

15. Foxwell AR, McIntyre P, Quinn H, Roper K, Clements MS Severe pertussis in infants: estimated impact of first vaccine dose at 6 versus 8 weeks in australia. Pediatr Infect Dis J. 2011;30(2):1613. http://dx.doi.org/10.1097/INF.ob013e3181f43906 PMID:20811313

16. Tozzi AE, Celentano LP, Ciofi degli Atti ML, Salmaso S. Diagnosis and management of pertussis. CMAJ. 
2005;172(4):509-15. http://dx.doi.org/10.1503/cmaj.1040766 PMID:15710944

17. Wymann MN, Richard JL, Vidondo B, Heininger U. Prospective pertussis surveillance in Switzerland, 1991-2006.

Vaccine. 2011;29(11):2058-65. http://dx.doi.org/10.1016/j. vaccine.2011.01.017 PMID:21251904

18. Nilsson L, Lepp T, von Segebaden K, Hallander H, Gustafsson L. Pertussis vaccination in infancy lowers the incidence of pertussis disease and the rate of hospitalisation after one and two doses: analyses of 10 years of pertussis surveillance. Vaccine. 2012;30(21):3239-47. http://dx.doi.org/10.1016/j. vaccine.2011.10.089 PMID:22094282

19. American Academy of Pediatrics. Pertussis (whooping cough) In: Pickering LK, Baker CJ, Long SS, McMillan JA, eds. Red Book: 2006 Report of the Committee on Infectious Diseases. 27 th ed. Elk Grove Village: American Academy of Pediatrics; 2006:498-520.

20. Bergquist SO, Bernander S, Dahnsjö H, Sundelöf B. Erythromycin in the treatment of pertussis: a study of bacteriologic and clinical effects. Pediatr Infect Dis J. 1987;6(5):458-61. http://dx.doi.org/10.1097/00006454198705000-00009 PMID:2885802 